\title{
The definition of dietary fiber - discussions at the Ninth Vahouny Fiber Symposium: building scientific agreement
}

\author{
John F. Howlett', Victoria A. Betteridge ${ }^{2}$, Martine Champ ${ }^{3}$, \\ Stuart A.S. Craig ${ }^{4}$, Agnes Meheust ${ }^{5 *}$ and Julie Miller Jones ${ }^{6}$
}

'Exponent International Limited, Pride Park, Derby, UK; ${ }^{2}$ Tate \& Lyle PLC, Sugar Quay, London, UK; ${ }^{3}$ CRNH, INRA, UMR 1280, Physiologie des Adaptations Nutritionnelles, Nantes, France; ${ }^{4}$ Danisco A/S, Tarrytown, NY, USA; ${ }^{5}$ ILSI Europe, Brussels, Belgium; ${ }^{6}$ Department of Family, Consumer and Nutritional Sciences, St. Catherine University, St. Paul, MN, USA

Abstract

A definition for dietary fiber was adopted in June 2009 by the Codex Alimentarius Commission based on the recommendation for endorsement of the Codex Committee on Nutrition and Foods for Special Dietary Uses (CCNFSDU) in November 2008. The definition listed three categories of carbohydrate polymers that are not hydrolyzed by the endogenous enzymes in the small intestine of humans. However, the definition left the inclusion of carbohydrates with degrees of polymerization (DP) in the range of 3 and 9 to the discretion of national authorities and left the 'physiological effect(s) of benefit to health' as undefined. The ILSI Europe and ILSI North America's committees on dietary carbohydrates organized a forum at the Ninth Vahouny Fiber Symposium in 2010 to discuss these implementation issues with the objective of building scientific consensus on how to resolve them. The results of this session are encouraging and indicated that the scientific community agrees on maintaining a worldwide consensus regarding the inclusion of non-digestible carbohydrates with $\geq$ DP3 as dietary fiber and on a core, non-exhaustive list of beneficial physiological effects that dietary fibers have. These results are consistent with previous worldwide agreements.

Keywords: dietary fiber; degree of polymerization; physiological effects; Vahouny Symposium; Codex Alimentarius

Received: 22 October 2010; Accepted: 23 October 20I0; Published: I November 2010

T he Ninth Vahouny Symposium on Dietary Fiber, which was held in Bethesda, Maryland from June $8-11$, 2010, was attended by more than 150 participants from academia, industry, and regulatory agencies. It was the first Vahouny Symposium to have been held since the adoption of a definition for dietary fiber by the Codex Alimentarius Commission in 2009 and, as such, presented an opportunity for issues surrounding the implementation of the Codex definition to be discussed by a body of scientific and regulatory experts pre-eminent in the field. Session 10 was jointly sponsored by the ILSI North America and ILSI Europe with this objective in mind. The session took the form of a workshop facilitated by Professor Julie Miller Jones of the Department of Family, Consumer and Nutritional Sciences at St. Catherine University, St. Paul, Minnesota and Dr. Martine Champ of the Nutritional Physiology
Unit of the National Institute for Agronomic Research (INRA), Nantes, France.

The goals of the session were to address critical aspects of the Codex definition of dietary fiber affecting its global implementation in a harmonized fashion and to provide a forum for experts in the field to address these impacts. This session was preceded by a session chaired by Tate $\&$ Lyle in which speakers had presented an overview of the Codex definition (Joanne Lupton, Texas A\&M University, College Station), an account of the methodology available for the analysis of dietary fiber in foods and beverages (Jon DeVries, General Mills, Minneapolis, $\mathrm{MN}$ ), an overview of European and international perspectives regarding the Codex definition (Wim Caers, Beneo-Group, Belgium), and the characterization of physiological benefits of dietary fiber (Joanne Slavin, University of Minnesota, St. Paul). 


\section{Proceedings of the session}

A summary of the implementation issues identified in the session was provided by Joanne Lupton to start the discussion. She reviewed the Codex definition for dietary fiber as adopted by the Codex Alimentarius Commission in 2009 (1), and noted that despite the adoption of the definition denoting a significant step forward for a global consensus on the nature and identity of dietary fiber, the following elements of the definition would benefit from further debate:

1. Footnote 2 to the definition, which leaves the inclusion of undigestible carbohydrates with degrees of polymerization (DP) in the range of 3 and 9 to the discretion of national authorities.

2. The absence of a list of beneficial physiological effects and appropriate criteria for their substantiation for the purpose of compliance with the definition.

3. The analytical methodology by which fiber in food is to be quantified.

Various aspects of these issues were the subject of discussion during the remaining part of Session 10 .

The exclusion/inclusion of carbohydrates with degrees of polymerization $(D P)$ in the range of 3 to 9

Debate about the exclusion or inclusion of carbohydrates with DPs in the range of 3 to 9 was focused on two major areas: (1) the lack of scientific support for differences in physiological effects between those oligomers with DP 3-9 and those with a higher DP and the absence of readily applicable methods that could clearly distinguish between them; and (2) the fact that the coexistence of regulations allowing two different standards for the same definition would undermine the validity of the definition.

All those who spoke regarding the physiological aspects argued that there was no basis for distinguishing between carbohydrates with a DP $\geq 10$ and those with DP $\leq 9$ because there were carbohydrates both above and below this cutoff point that exhibited one or more beneficial physiological effect(s) generally associated with fiber. The view was expressed that carbohydrates exhibiting beneficial physiological effects are distributed along a continuous spectrum of chain lengths with no clear differentiation at any particular DP.

Similarly, all those who spoke about methodological aspects were of the view that a universal cutoff point at a DP of 10 and above did not reflect methodological capability. Among those who spoke, there was a view that historically the cutoff point of DP $\geq 10^{1}$ had gained currency in the mistaken belief that it was consistently applicable to all carbohydrates in the frame for consideration as dietary fibers through precipitation in alcohol. In practice, this is not the case and methodology provides no reliable basis for imposing a distinction between carbohydrates with or without fiber-like properties on the basis of chain length alone.

In addition, many contributors to the debate were of the view that to provide a discretionary approach at the national level to excluding or including carbohydrate fractions within the scope of the definition was undesirable. For nutrition research and assessment, the absence of a common definition creates difficulties for the comparison of fiber intakes across different geographic regions and in the interpretation of studies assessing possible beneficial physiological effects where datasets are drawn from different regions. For consumers and food manufacturers, the application of different interpretations of what constitutes dietary fiber can result in a confusing nutrition messages for consumers, demand differences in food labeling of the same food marketed in different countries, and create difficulties for food manufacturers seeking to formulate products for a global market.

Nevertheless, if there had been a necessity for compromise in order to achieve agreement on a definition, then it would have been preferable to default to a position that included carbohydrates with DPs in the range of 3 to 9 within the body of the definition, with discretion included in the footnote for those who disagreed, rather than in the opposite manner as is currently in the Codex definition. If structured in this way, the default form of the definition would have been fully inclusive, more aligned with other existing definitions, and would have more accurately reflected the majority of opinion in the scientific community.

In summary, there was a strong consensus among contributors that there is no sound scientific basis for a cutoff point at a DP $\geq 10$. The difficulty of achieving a reconsideration of this issue within the Codex Alimentarius was acknowledged. It was suggested that the reaffirmation of the existing scientific agreement on the issue would provide better, practical support to national authorities in their implementation.

\section{Agreement on a list of beneficial physiological effects}

Initiating the discussion of beneficial physiological effects, Joanne Lupton drew attention to the three categories of dietary fiber differentiated within the Codex definition by their source: those occurring naturally in

\footnotetext{
${ }^{1}$ Prior to Codex discussion of a definition for dietary fiber, debate had centered on other cutoff points on the basis that oligomers below the cutoff DPs were soluble in $80 \%$ ethanol and those above were not. In practice, no clear cutoff point can be distinguished on the basis of solubility in $80 \%$ ethanol because solubility is also determined by the chemical nature of the constituent monosaccharides rather than the number of units alone and, therefore, the relationship between chain length and solubility in ethanol is imprecise.
} 
food as consumed; those obtained from food raw material by physical, enzymatic, or chemical means; and those that are synthetic in origin. Of the three categories, the definition requires that the latter two must be shown to have a physiological benefit to health, while for those occurring naturally in food as consumed, no such beneficial effect is required to be demonstrated. At the same time, the definition provides no description of what constitutes a beneficial physiological effect so, to the extent that this remains open to interpretation, it provides no clear indication of the qualifying features of fibers falling within the last two categories.

There is a diverse list of beneficial effects in common use in academia and by institutes, agencies, and authorities worldwide. Until the 2008 session of the Codex Committee on Nutrition and Foods for Special Dietary Uses (CCNFSDU) where the current definition was recommended for adoption, the Codex definition of dietary fiber itself had been presented in conjunction with an illustrative list of relevant beneficial physiological effects and had met with a clear majority of support among participating governments and observer organizations (2). The list was removed during the 2008 CCNFSDU session to simplify the definition (3), but the list's removal potentially leads to risks of higher levels of confusion as beneficial physiological effects are now open for different interpretations at the national level.

Dr. Lupton suggested that progress might be made by attempting to characterize effects in relation to three levels of agreement on the certainty of their validation:

1. Well-established beneficial effects

2. Probable beneficial effects

3. Possible beneficial effects

In this way, an agreed core list of beneficial effects could be drawn up to provide a working basis for the definition but, at the same time, the list could remain open to additions as emerging science provided sufficient validation.

During a discussion, the following physiological effects received support:

1. Reduced blood total and/or LDL cholesterol levels

2. Attenuation of postprandial glycemia/insulinemia

3. Reduced blood pressure

4. Increased fecal bulk/laxation

5. Decreased transit time

6. Increased colonic fermentation/short chain fatty acid production

7. Positive modulation of colonic microflora

8. Weight loss/reduction in adiposity

9. Increased satiety
Support was not unequivocal in every case. The occurrence of most of the effects was considered to be well established for fibers in general but the health impact of some effects was the subject of discussion. While in a few cases (reduced blood total cholesterol, reduced blood pressure) there were considered to be clear associations between the endpoints measured and the reduction of disease risk, in other cases (increased colonic fermentability, attenuation of postprandial glycemia/insulinemia, increased satiety) the relevance of the endpoint measured was considered by some to be indeterminate. It was also noted that there is an ongoing discussion about relevant methods and the interpretation of the magnitude of effect from the perspective of a contribution to health. Furthermore, it was pointed out that an agreement of the substantiation of the beneficial nature of any proposed effects is a case-by-case process.

The view was expressed that in considering beneficial effects in the context of a definition for dietary fiber and resultant nutrient content claims, it is important to keep in mind the consumption of fibers of all types. The total fiber content of the diet contributes several different effects simultaneously and the overall benefit, however achieved mechanistically, derives primarily from the fact that fiber is not digested in the small intestine and passes to the colon intact. The beneficial outcomes of individual fiber types in individual foods should be seen in terms of their contribution to the overall benefit achieved through their contribution to total dietary fiber intake as reflected in nutrient content claims. This is in contrast to health claims made in relation to individual components where the claim is product specific and requires substantiation on a case-by-case basis in relation to the individual food ingredient.

The distinction between these two circumstances is reflected in the nature of the claims made. In the case of fiber content claims, the primary consideration of beneficial effect is in relation to the total fiber content of the diet and the value to consumers of an awareness of the importance of maintaining an adequate intake of dietary fiber from a variety of sources. In the case of health claims, the consideration is entirely product-specific with the objective of making an on-pack claim, in a language understood by consumers for products containing the effective amount of the specific component.

Overall, there was enthusiasm for agreeing on a core list of beneficial physiological effects.

\section{Post-session survey}

At the suggestion of the audience, participants were invited to express their views by survey on whether or not carbohydrates with DPs in the range of 3 to 9 should be included in the definition of dietary fiber and, if a list of beneficial physiological effects were to be compiled, to 
Following the discussion held during the Joint ILSI North America - ILSI Europe session at the 9th Vahouny Fibre Symposium, Thursday 10 June 2010, do you agree with the following:

The Codex Alimentarius definition of dietary fibre should include carbohydrate polymers of DP 3 and above, which are not hydrolysed by the endogenous enzymes in the small intestine of humans and showing a physiological effect of benefit to health when pertaining to categories 2 and 3 (as described in the Codex Alimentarius definition of dietary fibre adopted in June 2009) as dietary fibres: YES/NO

In order to qualify as a dietary fibre the carbohydrate falling into the categories 2 and 3 of the Codex Almentarius definition (as adopted in June 2009) should demonstrate scientific evidence of at least one of, but not limited to, the following physiological effects listed below.

Which of the following physiological effects of benefit to health should be included on the list:

- reduction in blood total and/or LDL cholesterol levels YES/NO

- reduction in post-prandial blood glucose and/or insulinl evels YES/NO

- increased stool bulk and/or decreased gut transit time YES/NO

- fermentability by colonic microflora YES/NO

- other effect (s) YES/NO

Fig. 1. Survey circulated during the ILSI North America - ILSI Europe session.

express their preferences for the effects that should be included. Participants were asked to respond to the statements of the survey presented in Fig. 1, the results are displayed in Table 1.

Overall conclusions from the session and the survey There was overwhelming support among the participants during discussion in the session for the inclusion in the definition for dietary fiber of carbohydrate polymers with DPs in the range of 3 to 9 and of the responses to the survey, $86 \%$ were in favor of including them and 3\% were opposed. For reasons unknown, 11\% of respondents did not address the question. Taken together, the discussion during the session and the level of support shown by the survey indicates a convincing level of agreement among experts in the field that the science supports the inclusion of carbohydrate polymers with DPs in the range of 3 to 9 and provides a rationale for science-based decision making by national authorities in their implementation of the Codex definition.

In a discussion during the session, there was clear support for the establishment of a list of beneficial physiological effects associated with the consumption of dietary fiber. More than $80 \%$ (and, in the case of the first three, more than 95\%) of respondents to the survey indicated support for the inclusion of at least the following effects in the list:

Table 1. Seventy-five responses to the survey questionnaire were received and summarized

\begin{tabular}{lccc}
\hline & Positive answer & Negative answer & No answer \\
\hline Agree with the inclusion of DP 3-9 & $86.7 \%$ & $2.7 \%$ & $10.6 \%{ }^{\mathrm{a}}$ \\
Agree with physiological response: & & $1.3 \%$ & - \\
$-\quad$ Reduction in blood total and/or LDL cholesterol & $98.7 \%$ & $9.7 \%$ & $1.3 \%$ \\
- Reduction in postprandial blood glucose and/or insulin levels & $96 \%$ & - & $10.6 \%$ \\
$-\quad$ Increased stool bulk and/or decreased transit time & $98.7 \%$ & $82.7 \%$ & $69.3 \%$ \\
Proposed other physiological effects & $30.7 \%$ & & \\
\hline
\end{tabular}

${ }^{a}$ Three persons declined to answer the question DP 3-9 on grounds of insufficient information to allow a decision and five persons left the answer to the question concerning DP 3-9 blank. 
1. Reduction in blood total and/or LDL cholesterol

2. Reduction in postprandial blood glucose and/or insulin levels

3. Increased stool bulk and/or decreased transit time

4. Fermentability by colonic microflora

Almost a third of respondents to the survey $(30 \%)$ proposed the inclusion of effects additional to these four. This response would seem to argue strongly for the adoption of an open list of beneficial effects comprising in the first instance the above four listed functions and leaving open the possibility of adding other effects to the list as and when they achieve a similar level of acceptance as a result of developing science.

These results are consistent with several previous consensus documents. An international survey of fiber experts (4) found strong support for inclusion of oligosaccharides that are resistant to hydrolysis by human alimentary enzymes. Also, several recent regional expert opinions or definitions include oligosaccharides and/or a similar list of physiological effects (5-8).

\section{Acknowledgements}

J.H. had primary responsibility for final content; V.B, M.C., and J.J. chaired the scientific session discussion and provided substantive input into the paper content; A.M. and S.A.S.C. contributed substantially to writing and editing. All authors read and approved the final manuscript. The authors would like to particularly thank Dr. Jon deVries, Dr. Gunhild Kozianowski, Dr. Lisa Sanders, and Ms. Marie Latulippe for their contributions to this paper.

\section{Conflict of interest and funding}

J.F.H. is an employee of Exponent International Limited. J.F.H. received an honorarium for the drafting of this paper. V.A.B. is an employee of Tate \& Lyle PLC and a member of the ILSI Europe Dietary Carbohydrates Task Force. S.A.S.C. is an employee of Danisco A/S and a member of the ILSI North America Technical Committee on Carbohydrates. A.M. is an employee of ILSI Europe, which is partly funded by the food and related industries. J.M.J. is the scientific advisor of the ILSI North America Technical Committee on Carbohydrates and is a consultant for some not-for-profits and some food companies. The Vahouny symposium session and writing of this paper was sponsored by both the Dietary Carbohydrates Task Force of the European branch and the Carbohydrates Committee of the North American branch of the International Life Sciences Institute (ILSI). ILSI's programs are supported primarily by its industry membership. Industry members of the Dietary Carbohydrates Task Force of ILSI Europe are: AkzoNobel - National Starch Food Innovation, Cargill, Coca-Cola Europe, Colloïdes Naturels International, Danisco, Danone, Kellogg Europe, Kraft Foods, Nestlé, Premier Foods,
Südzucker/BENEO Group, Syral, and Tate \& Lyle. Industry members of the Carbohydrates Committee of ILSI North America are: Archer Daniels Midland Company, BENEO Group, Cargill Incorporated, The Coca-Cola Company, Corn Products International, Danisco USA Incorporated, Dr Pepper Snapple Group Incorporated, General Mills, The Hershey Company, Kellogg Company, Kraft Foods Incorporated, Mars Incorporated, McNeil Nutritionals, Mead Johnson Nutritionals, National Starch Food Innovation, Nestlé USA Incorporated, PepsiCo Incorporated, and Tate \& Lyle. The opinions expressed herein are those of the authors or discussion participants and do not necessarily represent the views of ILSI Europe or ILSI North America.

\section{References}

1. Codex Alimentarius 2010. Guidelines on nutrition labelling CAC/ GL 2-1985 as last amended 2010. Joint FAO/WHO Food Standards Programme, Secretariat of the Codex Alimentarius Commission, FAO, Rome.

2. Codex Alimentarius 2007. Report of the 29th session of the codex committee on nutrition and foods for special dietary uses, Bad Neuenahr-Ahrweiler, Germany, 12-16 November 2007, ALINORM 08/31/26.

3. Codex Alimentarius 2008. Report of the 30th session of the codex committee on nutrition and foods for special dietary uses, Cape Town, South Africa, 3-7 November 2008, ALINORM 09/32/26.

4. Lee SC, Prosky L. International survey on dietary fiber: definition, analysis and reference materials. J AOAC Int 1995; 78: 22-36.

5. American Association of Cereal Chemists (AACC). Definition of dietary fiber: report of the dietary fiber definition committee to the board of directors of the American association of cereal chemists. Cereal Foods World 2001; 6:112-26. At http:// www.aaccnet.org/news/pdfs/DFDef.pdf [cited 23 October 2010].

6. The National Academies of Science, Institute of Medicine (IOM). Dietary reference intakes for energy, carbohydrate, fiber, fat, fatty acids, cholesterol, protein, and amino acids. Washington, DC: National Academies Press; 2002, pp. 339-61. At http://www. nap.edu/openbook.php?record_id $=10490 \&$ page $=339$ [cited 23 October 2010].

7. Food Standards Australia New Zealand (FSANZ). Food Standards Australia New Zealand Code Issue 115, Standard 1.2.8 Nutrition Information Requirements. Page 2.

8. EFSA 2007. Statement of the scientific panel on dietetic products, nutrition and allergies on a request from the Commission related to dietary fibre (Request No. EFSA-Q-2007-121). Expressed on 6 July 2007 at its 17 th plenary meeting corresponding to item 10.1 of the Agenda. At http://www.efsa.europa.eu/en/scdocs/scdoc/ 1060.htm [cited 23 October 2010].

\footnotetext{
*Agnes Meheust

ILSI Europe a.i.s.b.l

av. E. Mounier 83, Box 6

BE- 1200 Brussels, Belgium

Tel: $+32(0) 277 \mid 00$ | 4

Fax: $+32(0) 27620044$

Email:publications@ilsieurope.be
} 